\title{
THE COMPLEMENT OF A FINITELY GENERATED DIRECT SUMMAND OF AN ABELIAN GROUP
}

\author{
P. M. COHN
}

1. In his recent monograph on abelian groups [1], Kaplansky raises the following question:

If $F$ is a finitely generated abelian group and $G, H$ are any abelian groups such that $F \oplus G \cong F \oplus H$, are $G$ and $H$ isomorphic?

We shall answer this question affirmatively. In the first place we can reduce the problem to the case where $F$ is cyclic of infinite or prime power order. For suppose that the answer has been obtained in this case, and let $F$ be any finitely generated abelian group. Then $F$ is a direct sum of a finite number of cyclic groups, each of infinite or prime power order, say

$$
F=F_{1} \oplus F_{2} \oplus \cdots \oplus F_{k}
$$

and

$$
F_{1} \oplus \cdots \oplus F_{k} \oplus G \cong F_{1} \oplus \cdots \oplus F_{k} \oplus H .
$$

We use induction on $k$. By the result for the cyclic case, we may cancel $F_{1}$ and obtain

$$
F_{2} \oplus \cdots \oplus F_{k} \oplus G \cong F_{2} \oplus \cdots \oplus F_{k} \oplus H,
$$

and therefore $G \cong H$, by induction.

2. It remains to deal with the cyclic case. By identifying $F \oplus G$ and $F \oplus H$ we may restate the assertion as follows: If $E$ is an abelian group which may be written as a direct sum in two ways, $E=A \oplus G$ $=B \oplus H$, where $A$ and $B$ are cyclic subgroups of $E$ of the same order $\infty$ or $p^{n}$, then $G \cong H$.

3. We first dispose of the case where $A$ and $B$ are infinite. Let $G \cap H=D$, then $G / D \cong G / G \cap H \cong(G+H) / H \cong$ subgroup of $E / H \cong B$. Thus $G / D$ is infinite cyclic or 0 . Similarly for $H / D$. If $G / D$ and $H / D$ are both zero, then $G=D=H$, so suppose that $G / D$ is infinite cyclic. Choose $u \in G$ such that the residue-class $D+u$ generates $G / D$, and let $U$ be the subgroup of $G$ generated by $u$. Then $G=U \oplus D$, as is

Received by the editors June 16, 1955.

1 Editor's note: A paper by E. A. Walker has been received whose contents are similar to those of the paper published here. $\mathrm{Cf}$. also the paper of E. A. Walker, to be published in a later issue. 
easily seen. Thus $E=A \oplus U \oplus D=B \oplus H$, and $D \subseteq H$. Taking quotients by $D$ we obtain $A \oplus U \cong B \oplus H / D$, hence $H / D$ is infinite cyclic; any representative in $H$ of a generator $\bmod D$ generates an infinite cyclic group $\mathrm{V}$ which satisfies $H=V \oplus D$. Thus $G=U \oplus D \cong V \oplus D=H$.

4. Now suppose that $A$ and $B$ are finite, of order $p^{n}$ say, and are generated by $a$ and $b$ respectively. We show first that there exists an element $u$ of order $p^{n}$ such that no nonzero multiple of $u$ belongs to $G$ or $H$. If neither $a$ nor $b$ satisfies this condition, then since no nonzero multiple of $a$ lies in $G$, we must have $p^{n-1} a \in H$, and similarly $p^{n-1} b \in G$. Put $u=a+b$, then $p^{n} u=0$, while $p^{n-1} u \equiv p^{n-1} a \neq 0(\bmod G)$ and $p^{n-1} u \equiv p^{n-1} b \not \equiv 0(\bmod H)$. Thus there is always such an element $u$. Let $U$ be the subgroup generated by $u$. Then from the definition, $U \cap G=0$, while $U+G / G \cong U / U \cap G \cong U$. Thus $G$ has the index $p^{n}$ in $U+G$ and the same index in $E$, which contains $U+G$. Therefore $E=U+G$, and in fact $E=U \oplus G$, because $U \cap G=0$. Similarly $E=U \oplus H$, and $G \cong E / U \cong H$.

\section{REFERENCE}

1. I. Kaplansky, Infinite abelian groups, Ann Arbor, 1954.

THE UNIVERSITY, MANCHESTER, ENGLAND 